\title{
Uniqueness of Solutions to Schrödinger Equations on Complex Semi-Simple Lie Groups
}

by

\author{
Sagun Chanillo
}

\begin{abstract}
In this note we study the time dependent Schrödinger equation on Complex semisimple Lie Groups. We show that if the initial data is a bi-invariant function that has sufficient decay and the solution has sufficient decay at another fixed value of time, then the solution has to be identically zero for all time. We also derive Strichartz and decay estimates for the Schrödinger equation. Our methods also extend to the wave equation. On the Heisenberg group we show that the failure to obtain a parametrix for our Schrödinger equation is related to the fact that geodesics project to circles on the contact plane at the identity.
\end{abstract}

Dedicated to U. B. Tewari on his Retirement

MSC(Subj. Classification): 43A85

Keywords: Schrödinger Equation, Uniqueness, Strichartz Estimates, Complex Lie Groups, Heisenberg group.

Running Head: Schrödinger's Equation on Complex Lie Groups 


\section{$\S 1$. Introduction:}

Let $G$ denote a Lie group. We are concerned here with the initial value problem for the time dependent Schrödinger eqn.,

$$
-i u_{t}=\Delta u,\left.u\right|_{t=0}=f(x), x \in G
$$

$\Delta$ will denote the Laplace-Beltrami operator with respect to an appropriate $G$ invariant metric. Our main aim is to obtain conditions on $f$ which will guarantee that the solution $u(x, t)$, vanishes identically for all $t>0$. The results that we have may be all viewed as consequences of a well-known theorem of Hardy on the Euclidean Fourier transform, which is a form of the uncertainty principle. The Hardy theorem has been extended to the Lie group setting by various authors, see [2], [9]. However, we do not need any Lie group version of the Hardy theorem, the original Euclidean version will suffice in our applications to Lie groups. We plan to extend our results to the real semi-simple Lie groups and to obtain versions on the Heisenberg group later. On nilpotent groups there is a serious difficulty since the geodesics project to circles on the contact plane and the existence of such closed loops creates difficulties in writing down a parametrix for the solution operator to (1.1), see section 3. Our results may be viewed as a statement that a concentrated wave packet at initial time will spread out and if still remains concentrated, it must be trivial. Lastly in Sec 4. we derive decay estimates and Strichartz estimates for bi-invariant solutions to the Schrödinger equation on complex semi-simple groups. The methods extend to the wave equation on complex Lie groups.

We wish to thank Roe Goodman for reading our paper and offering constructive criticism. The research for this paper was supported in part by an NSF grant DMS0600971.

\section{$\S 2$. The Uniqueness theorem}

We recall the theorem of Hardy. Let the Fourier transform be defined as,

$$
\hat{f}(\xi)=\int_{R^{n}} e^{-i<x, \xi>} f(x) d x .
$$

Theorem 1: (Hardy [4]) Let $f(x)$ satisfy $|f(x)| \leq C e^{-a|x|^{2}}$. Furthermore, assume, $|\hat{f}(\xi)| \leq$ $B e^{-b|\xi|^{2}}$. If $4 a b>1$, then $f \equiv 0$.

We shall now derive from this theorem a uniqueness theorem for solutions to (1.1) when $G=R^{n}$.

Theorem 2: Let us consider the initial value problem for $u(x, t)$,

$$
-i u_{t}=\Delta u,\left.u\right|_{t=0}=f(x)
$$

Assume that $|f(x)| \leq A e^{-a|x|^{2}}$, and $\left|u\left(x, t_{0}\right)\right| \leq B e^{-b|x|^{2}}$. If $16 a b t_{0}^{2}>1$, then $u(x, t) \equiv$ 0 , for all $t \geq 0$. 
Proof: We recall that using the fundamental solution of (1.1), we may write,

$$
u(x, t)=\frac{c_{n}}{t^{n / 2}} \int_{R^{n}} e^{i \frac{|x-y|^{2}}{4 t}} f(y) d y .
$$

We may re-write the last identity as,

$$
u(x, t)=\frac{c_{n} e^{i \frac{|x|^{2}}{4 t}}}{t^{n / 2}} \int_{R^{n}} e^{-i<\frac{x}{2 t}, y>} e^{i \frac{|y|^{2}}{4 t}} f(y) d y
$$

Now set $h(y)=e^{i \frac{|y|^{2}}{4 t}} f(y)$. Then from (2.1) we get,

$$
u(x, t)=\frac{c_{n} e^{i \frac{|x|^{2}}{4 t}}}{t^{n / 2}} \hat{h}\left(\frac{x}{2 t}\right) .
$$

Now we take $t=t_{0}$ and apply Hardy's theorem. From the hypothesis on $u\left(x, t_{0}\right)$, we have,

$$
\left|\hat{h}\left(\frac{x}{2 t_{0}}\right)\right| \leq B e^{-b|x|^{2}}
$$

Thus,

$$
|\hat{h}(x)| \leq B e^{-4 b t_{0}^{2}|x|^{2}}
$$

Clearly $|h(x)| \leq C e^{-a|x|^{2}}$. Since $16 a b t_{0}^{2}>1$, Hardy's theorem applies and we conclude $h \equiv 0$. It then follows that $f \equiv 0$ and hence $u(\cdot, t) \equiv 0$ for all $t$.

We now wish to extend Theorem 2 to a complex semi-simple Lie group. We need to introduce some notation.

Let $G$ denote a complex, connected semi-simple Lie group. Let $K$ denote a fixed maximal compact sub-group of $G$. Let $\mathcal{G}$ and $\mathcal{K}$ denote the Lie algebras of $G$ and $K$ respectively. Let $B$ denote the Cartan-Killing form on $\mathcal{G}$, and the Cartan decomposition is given by $\mathcal{G}=\mathcal{K} \oplus \mathcal{P}$. The restriction of $B$ to $\mathcal{P} \times \mathcal{P}$ is strictly positive definite and hence defines a norm. Let $\mathcal{A}$ be a fixed maximal Abelian subspace of $\mathcal{P}$. Let $\Sigma$ denote the set of non-zero roots corresponding to the pair $(\mathcal{G}, \mathcal{A})$, and $\Sigma_{+}$the set of positive roots $\alpha$ for some ordering. $W$ will denote the Weyl group associated to $\Sigma$. Let $\mathcal{A}_{+}$be the positive Weyl chamber associated to $\Sigma$. Let $A$ denote the analytic sub-group with Lie algebra $\mathcal{A}$. We have the map, $\exp : \mathcal{A} \rightarrow A$. Likewise we have, $A_{+}=\exp \left(\mathcal{A}_{+}\right)$. Then one has the polar decomposition,

$$
G=K \overline{A_{+}} K
$$

The Haar measure on $A$ can then be written for this polar decomposition by a formula of Harish-Chandra [5] as,

$$
d x=\left(\sum_{s \in W}(\operatorname{det} s) e^{s \rho(H)}\right)^{2} d H=\phi(H)^{2} d H, H \in \mathcal{A} .
$$


As usual $\rho=\frac{1}{2} \sum_{\Sigma_{+}} \alpha$. The spectral variables are elements of $\mathcal{A}^{\star}$ and will be denoted by $\lambda$. On $G / K$ we have a $G$ invariant Riemannian metric obtained through the Killing form and we can form a Laplace-Beltrami operator $\Delta$ using this metric. See [7]. Note now, that there is a unique element $H_{\lambda} \in \mathcal{A}$, such that,

$$
\lambda(H)=B\left(H_{\lambda}, H\right), H \in \mathcal{A} .
$$

The norm of elements $H \in \mathcal{A}$ will be denoted by,

$$
|H|^{2}=B(H, H)
$$

Lastly a function $f$ on $G$ will be said to be $K$ bi-invariant if and only if,

$$
f\left(k_{1} a k_{2}\right)=f(a), a \in A
$$

From the polar decomposition we may view the function as only depending on its values on $A_{+}$, or by using the inverse exponential map we may also view $f$ as a complex valued function on $\mathcal{A}$ solely determined by its values on $\mathcal{A}_{+}$. We are ready to state our theorem.

Theorem 3: Let $G$ denote a connected, complex, semi-simple Lie group. Let $f$ be a bi-invariant function such that,

$$
|f(H)| \leq A e^{-a|H|^{2}} .
$$

Consider the initial value problem,

$$
-i u_{t}=\Delta u,\left.u\right|_{t=0}=f
$$

Then $u$ is also bi-invariant. Furthermore if

$$
\left|u\left(H, t_{0}\right)\right| \leq B e^{-b|H|^{2}},
$$

and $16 a b t_{0}^{2}>1$, then necessarily $u \equiv 0$ for all $t \geq 0$.

Proof: The proof requires the use of well-known facts about spherical functions. First we need to recall Theorem 5.7, p.432, [7] which states that on a complex semi-simple group, the elementary spherical functions are given by(where $\phi(H)$ is as in $(2.3))$,

$$
\phi_{\lambda}(H)=c(\lambda) \frac{\sum_{s \in W}(\operatorname{det} s) e^{i s \lambda(H)}}{\phi(H)}
$$

Here $c(\lambda)$ is the celebrated c-function of Harish-Chandra [5,6], [3]. Furthermore we also have from [7] that,

$$
\Delta \phi_{\lambda}(H)=-\left(|\lambda|^{2}+|\rho|^{2}\right) \phi_{\lambda}(H)
$$


The spherical transform of $f$ is given by,

$$
\hat{f}(\lambda)=\int_{\mathcal{A}} \phi_{-\lambda}(H) f(H) \phi^{2}(H) d H .
$$

Thus the solution of our initial value problem in view of (2.6) is,

$$
u(H, t)=\int_{\mathcal{A}^{\star}} e^{-i t\left(|\lambda|^{2}+|\rho|^{2}\right)} \phi_{\lambda}(H) \hat{f}(\lambda)|c(\lambda)|^{-2} d \lambda .
$$

We insert (2.5), (2.7) into (2.8) to get,

$$
\begin{gathered}
u\left(H_{1}, t\right) \phi\left(H_{1}\right)=\int_{\mathcal{A}}\left(\int_{\mathcal{A}^{\star}} e^{-i t\left(|\lambda|^{2}+|\rho|^{2}\right)}\left(\sum_{s \in W}(\operatorname{det} s) e^{i s \lambda\left(H_{1}\right)}\right)\left(\sum_{s^{\prime} \in W}\left(\operatorname{det} s^{\prime}\right) e^{-i s^{\prime} \lambda\left(H_{2}\right)}\right) d \lambda\right) \\
f\left(H_{2}\right) \phi\left(H_{2}\right) d H_{2} .
\end{gathered}
$$

The inner integral in $\lambda$ may be re-written in view of (2.4) as,

$$
\sum_{s, s^{\prime}}(\operatorname{det} s)\left(\operatorname{det} s^{\prime}\right) e^{-i t|\rho|^{2}} e^{i \frac{\left|s H_{1}-s^{\prime} H_{2}\right|^{2}}{4 t}} \int_{\mathcal{A}} e^{-i t\left|H_{\lambda}+\frac{1}{2 t}\left(s H_{1}-s^{\prime} H_{2}\right)\right|^{2}} d H_{\lambda}
$$

Evaluating the integral in $(2.10)$ we get from $(2.9),(l=\operatorname{dim} \mathcal{A})$,

$$
u\left(H_{1}, t\right) \phi\left(H_{1}\right)=c_{l} \frac{e^{-i t|\rho|^{2}}}{t^{l / 2}} \int_{\mathcal{A}}\left(\sum_{s, s^{\prime}}(\operatorname{det} s)\left(\operatorname{det} s^{\prime}\right) e^{i \frac{\left|s H_{1}-s^{\prime} H_{2}\right|^{2}}{4 t}}\right) f\left(H_{2}\right) \phi\left(H_{2}\right) d H_{2} .
$$

Set $g\left(H_{2}\right)=f\left(H_{2}\right) \phi\left(H_{2}\right)$. Now note that because $f$ is bi-invariant and $\phi(H)$ is odd under the action of the Weyl group, $g(s H)=(\operatorname{det} s) g(H)$. We proceed to re-write $(2.11)$ as in the Euclidean case and we will make use of the Euclidean Fourier transform. Re-writing (2.11) we get,

$$
u\left(H_{1}, t\right) \phi\left(H_{1}\right)=c_{l} \frac{e^{-i t|\rho|^{2}} e^{i \frac{\left|H_{1}\right|^{2}}{4 t}}}{t^{l / 2}} \sum_{s, s^{\prime}}(\operatorname{det} s)\left(\operatorname{det} s^{\prime}\right) \int_{R^{l}} e^{-i B\left(\frac{s s^{\prime} H_{1}}{2 t}, H_{2}\right)} e^{i \frac{\left|H_{2}\right|^{2}}{4 t}} g\left(H_{2}\right) d H_{2} .
$$

Here $l$ is the rank of $G$. Set

$$
R\left(H_{2}\right)=e^{i \frac{\left|H_{2}\right|^{2}}{4 t}} g\left(H_{2}\right)
$$

Then (2.12) states,

$$
u\left(H_{1}, t\right) \phi\left(H_{1}\right)=c_{l} \frac{\left.e^{-i\left(t|\rho|^{2}-\frac{\left|H_{1}\right|^{2}}{4 t}\right.}\right)}{t^{l / 2}} \sum_{s, s^{\prime}}(\operatorname{det} s)\left(\operatorname{det} s^{\prime}\right) \hat{R}\left(\frac{s s^{\prime} H_{1}}{2 t}\right)
$$


Since $R(H)$ is odd under reflection by the Weyl group, we finally can write $(2.14)$ as,

$$
u\left(H_{1}, t\right) \phi\left(H_{1}\right)=c_{l}|W|^{2} \frac{e^{-i\left(t|\rho|^{2}-\frac{\left|H_{1}\right|^{2}}{4 t}\right)}}{t^{l / 2}} \hat{R}\left(\frac{H_{1}}{2 t}\right) .
$$

Noting further from (2.3) that $|\phi(H)| \leq c e^{c|H|}$, we see right away from (2.15) and the hypothesis that at $t=t_{0}$,

$$
\left|\hat{R}\left(H_{1}\right)\right| \leq c e^{-4 b^{\prime} t_{0}^{2}\left|H_{1}\right|^{2}}, b^{\prime}<b
$$

and also,

$$
\left|R\left(H_{2}\right)\right| \leq c e^{-a^{\prime}\left|H_{2}\right|^{2}}, a^{\prime}<a .
$$

Thus by Theorem 1 , we again conclude $R \equiv 0$. This implies $f \equiv 0$ and hence the theorem follows.

Lemma 1: The results of Theorems 2 and 3 are sharp. One cannot relax the condition $16 a b t_{0}^{2}>1$.

Proof: We only display the proof for $R^{n}$. For theorem 3 one can check the validity of the lemma by doing an explicit computation on $S L(2, C)$. For $R^{n}$, choose initial data,

$$
f(x)=e^{-|x|^{2}-i \frac{|x|^{2}}{4}}
$$

An elementary computation using (2.1) shows that,

$$
u(x, 1)=c_{n} e^{\frac{i|x|^{2}}{4}} e^{-\frac{|x|^{2}}{16}}
$$

Thus we have $16 a b t_{0}^{2}=1$ and uniqueness fails.

\section{$\S 3$. The Heisenberg Group.}

We may ask the uniqueness question above for the sub-Laplacian $\Delta_{b}$ on the Heisenberg group. That is we consider the Schrödinger equation,

$$
i u_{t}=\Delta_{b} u,\left.u\right|_{t=0}=f(x)
$$

However there is a difficulty in writing the fundamental solution to this operator due to the presence of closed loops in the contact plane. To see this we proceed heuristically. First of all the fundamental solution to the heat equation on the Heisenberg group is given by,

$$
K(x, u, \xi, t)=\int_{-\infty}^{\infty} e^{-i \lambda \xi} e^{-t \lambda^{2}} \frac{\lambda}{\sinh (\lambda t)} e^{-\frac{1}{4} \lambda \operatorname{coth}(\lambda t)\left(x^{2}+u^{2}\right)} d \lambda
$$


where the Heisenberg group is viewed as $R^{3}$ and points on it written as $(x, u, \xi)$. To try to get a solution operator for (3.1), we perform a change of variables in (3.2) by letting, $t \rightarrow-i t$. The integrand in (3.2) becomes,

$$
\frac{\lambda}{\sin (\lambda t)} e^{-\frac{i}{4} \lambda \cot (\lambda t)\left(x^{2}+u^{2}\right)}
$$

Thus we note that the putative solution operator for fixed $t$ is singular at $\lambda=k \pi / t$. In fact (3.3) converges to a Dirac delta at $u=x=0$ as $\lambda \rightarrow k \pi / t$. This phenomena is attributable to the geodesics of the Heisenberg group projecting onto circles(closed loops) in the contact plane at the origin and having a cut-locus at $k \pi / t$. The geodesics on the Heisenberg group are well-known and a formula is found in [8]. They are given by,

$$
\begin{gathered}
x(s)=\frac{\cos \beta(1-\cos (t s))+\sin \beta \sin (t s)}{t} \\
u(s)=\frac{-\sin \beta(1-\cos (t s))+\cos \beta \sin (t s)}{t} \\
\xi(s)=2 \frac{t s-\sin (t s)}{t^{2}}
\end{gathered}
$$

Now notice the geodesics are not closed but their projection into the $x-u$ plane, the contact plane, are circles given by,

$$
\left(x(s)-\frac{\cos \beta}{t}\right)^{2}+\left(u(s)+\frac{\sin \beta}{t}\right)^{2}=\frac{1}{t^{2}} .
$$

The cut-locus emerges exactly at a distance of $k \pi / t$ from $u=x=0$ along the circle of radius $1 / t$, and it is exactly there the integrand of the solutions operator has its singularities. A general construction of the heat kernel on CR manifolds in [1] exhibits the same phenomena.

\section{§4. Strichartz Estimates and Decay estimates on Complex Lie Groups}

We will use the results of our computation in Sec. 2 on complex semi-simple groups to obtain various estimates on the solution $u(H, t)$. The estimates fall into two categories. One where we integrate over a fixed time slice and another where we integrate over both space and time, the Strichartz estimates. We have,

Theorem 4: Under the assumptions of theorem 3, the solution $u(H, t)$ for bi-invariant initial data $f$ satisfies,

$$
\left\|u|\phi|^{1-\frac{2}{q}}\right\|_{L^{q}(G)} \leq c t^{-l\left(\frac{1}{p}-\frac{1}{2}\right)}|| f|\phi|^{1-\frac{2}{p}} \|_{L^{p}(G)}, 1 \leq p \leq 2, \frac{1}{p}+\frac{1}{q}=1 .
$$

Assume that,

$$
-i u_{t}-\Delta u=\psi(H, t),\left.u\right|_{t=0}=f
$$


where $\psi$ is also bi-invariant for every fixed $t$. Then for $p=2(l+2) /(l+4), q=2(l+2) / l$, we have,

$$
\left\|u|\phi|^{1-\frac{2}{q}}\right\|_{L^{q}(G \times(0, \infty))} \leq c\left(\|f\|_{L^{2}(G)}+\left\|\psi|\phi|^{1-\frac{2}{p}}\right\|_{L^{p}(G \times(0, \infty))}\right)
$$

Proof: The proofs follow from the identity (2.11). It follows from (2.11) that the function $u(H, t) \phi(H)$ is obtained by applying the Euclidean fundamental solution of the Schrödinger equation to the data given by $g(H)$. Thus from the Euclidean estimates,

$$
\|u \phi\|_{L^{q}\left(R^{l}\right)} \leq c t^{-l\left(\frac{1}{p}-\frac{1}{2}\right)}\|g\|_{L^{p}\left(R^{l}\right)}
$$

where $p, q$ is as in part $(a)$ above. Re-writing the last inequality using (2.3) we get part (a) of our theorem.

A similar computation as in Cor. 1, in [10] gives part (b).

Remark: The methods of Theorem 4 and [10] also extend to the wave equation on complex semi-simple Lie groups with bi-invariant functions as data. Strichartz and decay estimates are easily obtained by following the methods developed in section 2 and theorem 4 . 


\section{References}

[1] Beals, R., Greiner, P. C. and Stanton, N., The Heat Equation on a CR Manifold, J. Differential Geom., 20, (1984), 343-387.

[2] Folland, G. B., and Sitaram, A., The uncertainty Principles: A Mathematical Survey, J. Fourier Analysis Appl. 3, (1997), 207-238.

[3] Gindikin, S. and Karpelevic, F. I., Plancherel Measure of Riemannian Symmetric spaces of non-positive curvature, Dokl. Akad. Nauk. SSSR, 145, (1962), 252-255.

[4] Hardy, G. H., A theorem concerning Fourier transforms, J. London Math. Soc., 8, (1933), 227-231.

[5] Harish-Chandra, Spherical Functions on a Semi-simple Lie Group I, Amer. J. of Math., 80, (1958), 241-310.

[6] Harish-Chandra, Spherical functions on a Semi-simple Lie group II, Amer. J. of Math., 80, (1958), 553-613.

[7] Helgason, S., Groups and Geometric Analysis-Integral Geometry, Invariant Differential Operators and Spherical Functions, Academic Press, New York, 1984.

[8] Monti, R., Some properties of Carnot-Caratheodory balls in the Heisenberg group, Rend. Math. Acc. Lincei, 11, s.9, (2000), 155-167.

[9] Sitaram, A., and Sundari, M., An analogue of Hardy's theorem for very rapidly decreasing functions, Pacific J. Math., 177, (1997), 187-200.

[10] Strichartz, R. S., Restrictions of Fourier Transforms to Quadratic Surfaces and Decay of Solutions to Wave Equations, Duke Math. J. 44(3), (1977), 705-714.

Deptt. of Math.,

Rutgers University, 110 Frelinghuysen Rd., Piscataway, NJ 08854

USA

e-mail address: chanillo@math.rutgers.edu 